UNITED STATES DEPARTMENT OF THE INTERIOR

GEOLOGICAL SURVEY

\title{
MSTRACK -- A computerized tracking system for manuscripts written in Paradox for the IBM DC and compatibles
}

by

Wendy S. Speckman*

Open-File Report 91-443-A

Diskette Version 91-443-B

Although program tests have been made, no guarantee (expressed or implied) is made by the author or the U.S. Geological Survey regarding program correctness, accuracy, or proper execution on all computer systems. This report is preliminary and has not been reviewed for conformity with U.S. Geological Survey editorial standards. Any use of trade names is for descriptive purposes only and does not imply endorsement by the U.S. Geological Survey.

* U.S. Geological Survey, DFC, Box 25046, MS 973, Denver, CO 80225 
TABLE OF CONTENTS

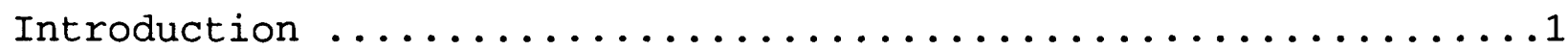

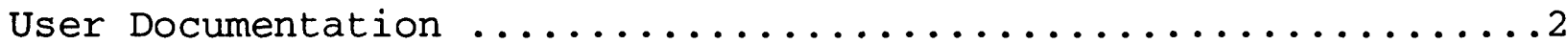

Description of main menu options $\ldots \ldots \ldots \ldots \ldots \ldots \ldots \ldots \ldots$

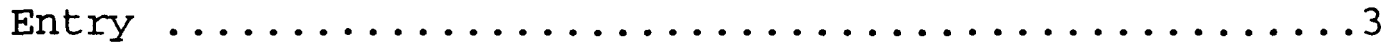

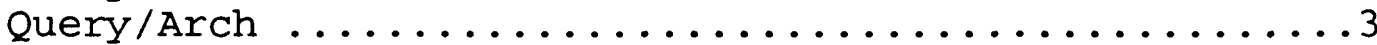

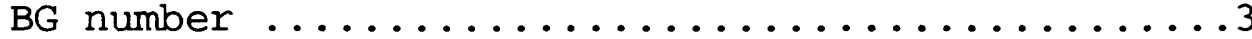

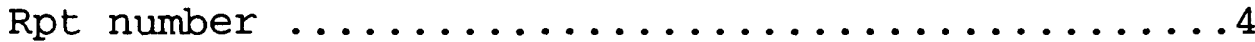

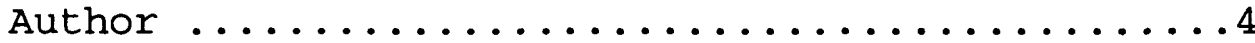

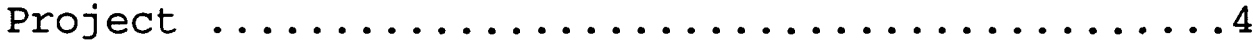

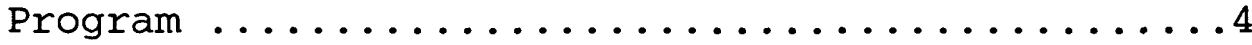

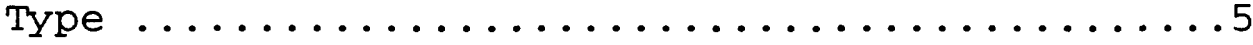

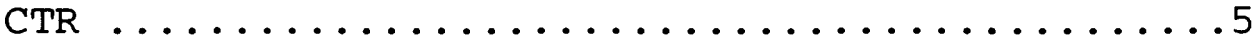

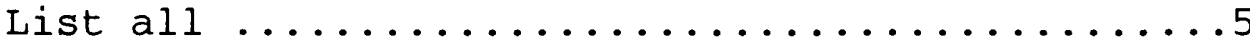

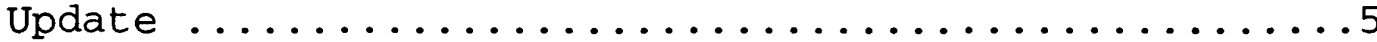

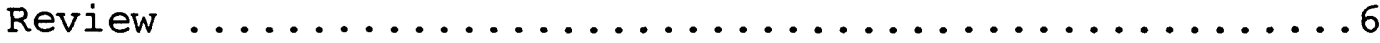

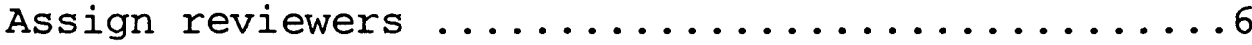

List reviewers ......................

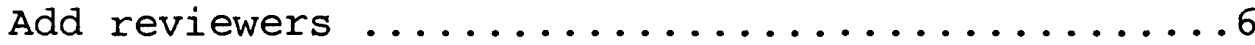

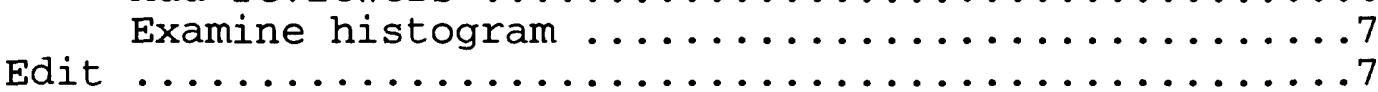

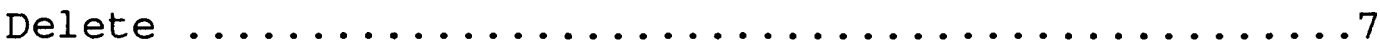

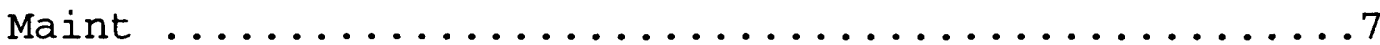

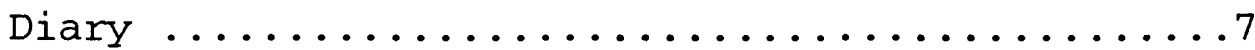

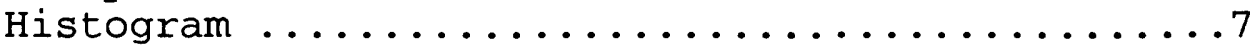

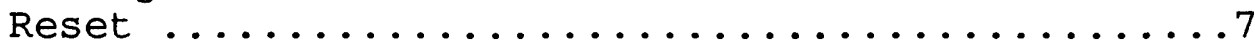

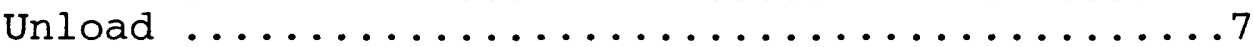

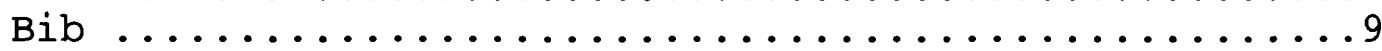

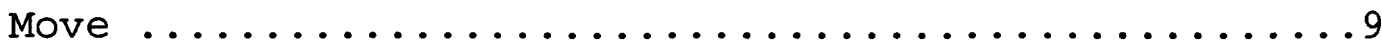

Programmer Documentation.........................

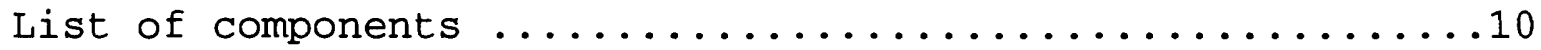

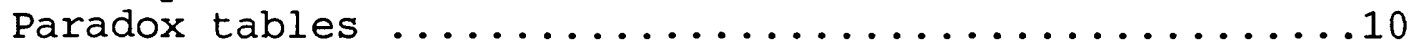

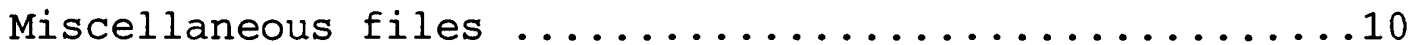

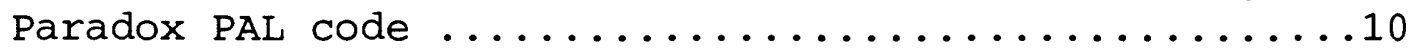

Description of modules contained in TRKLIB.SC .........12

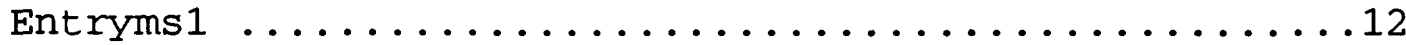

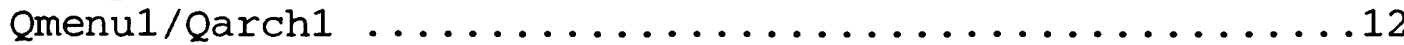

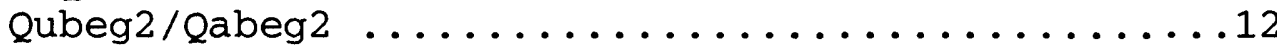

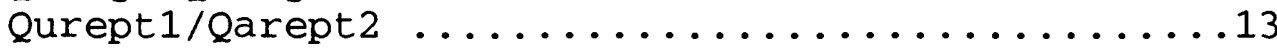

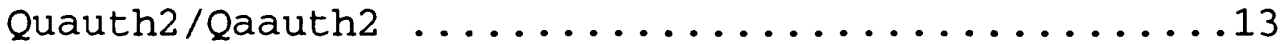

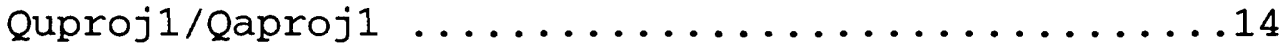

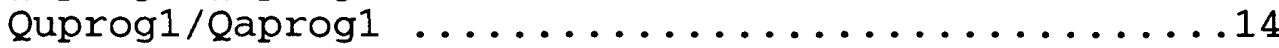

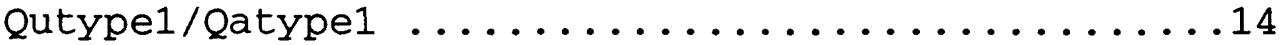

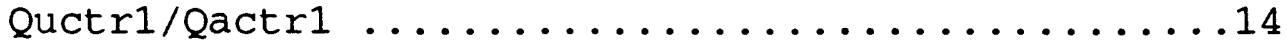

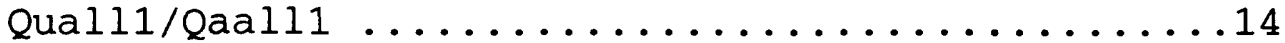

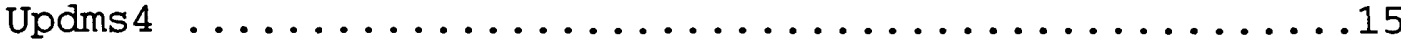




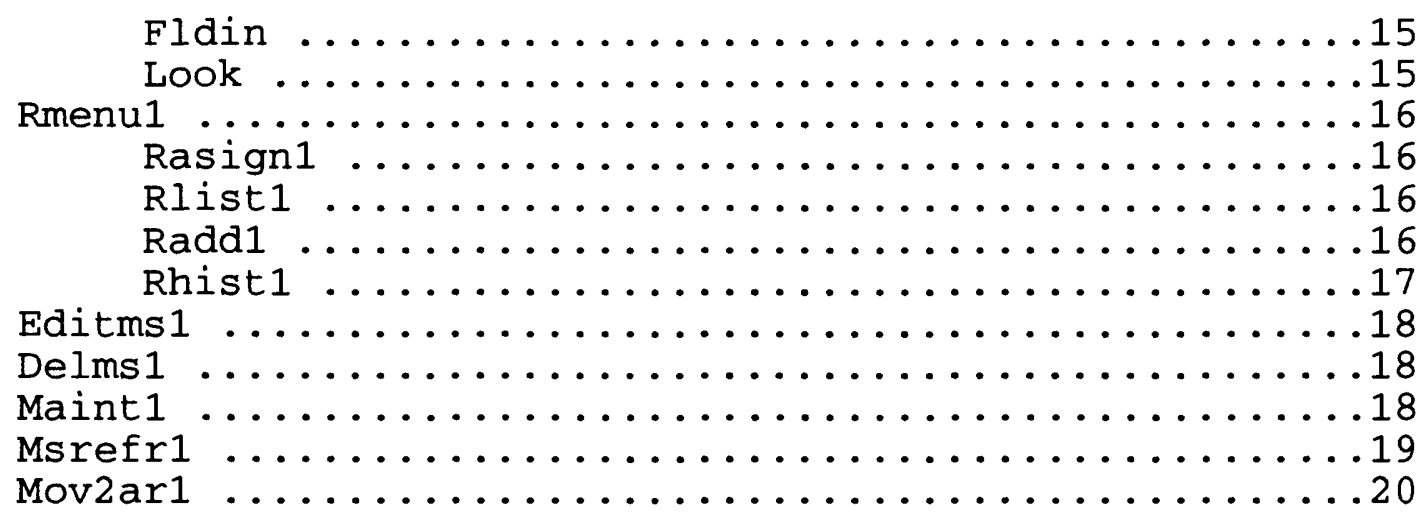

Appendix A. Paradox table structures .................. A1

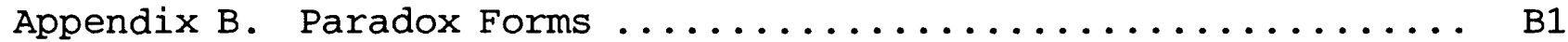

Appendix C. Paradox Reports ....................... C1 


\section{Introduction}

MSTRACK is a computerized tracking system for manuscripts written by members of the Branch of Geochemistry. Members of the Technical Publications Support Group in the Branch of Geochemistry are the primary users of this system. They use MSTRACK to record the movement of a manuscript through the review and publication process. MSTRACK operates on a personal computer running DOS and requires PARADOX 3.0 or later.

The database is in two parts: (1) an active database consisting of manuscripts currently being routed through the review and publication process, and (2) an archival database consisting of manuscripts that have been published or are no longer active. The archival system is kept to be used for Branch bibliographies.

To operate the manuscript tracking system, the user must change working directory into the directory that contains the database and type MSTRACK. This invokes a DOS BAT file which, in turn, invokes PARADOX with the proper script files.

The source code for the MSTRACK system is included as Report 91443-B on floppy disk. The disk contains MSTRACK. BAT (batch file to invoke Paradox with correct script files), MENUMS1.SC

(displays main menu), and TRKLIB.SC (contains source code for all procedures used and creates TRACKLB. LIB when PLAYED in Paradox).

Hard copies of the table structures are found in Appendix A. Hard copies of the Forms are found in Appendix B. Hard copies of the Reports are found in Appendix C. 


\section{User Documentation}

When the user types MSTRACK, the main menu is displayed as follows:

Entry: Initial Entry of manuscript into database

Query: List information about manuscripts

Update: Update location of manuscript, by BG number

Review: Reviewer manipulation

Edit: Edit information about a manuscript, not location

Delete: Deletes manuscript specified by BG number from database

Maint: Diary, histogram, reset, and unload functions

Bib: Enter complete bibliographic reference

Arch: Query the archival database

Move: Move manuscript from ACTIVE database to ARCHIVAL

Leave: Leave the application

All prompts for the BG (Branch of Geochemistry) number throughout the tracking system have the following format: "SUBMENU LOCATION: Enter BG number:" (example: "LOCATION UPDATE: Enter BG number: "). This is done so that the user can always tell in which sub-system they are working. The BG number should always be entered in the form FY-999 (example 1-012). Pressing the ENTER key at this prompt returns the user to the menu.

Every action that changes the database is recorded in a "diary file" named DIARYMS. The diary file is an ASCII file that is a chronological log of activity in the database. The entry made in DIARYMS has the date that the actual changes were made to the database, not the date that was entered into the database. 


\section{DESCRIPTION OF MAIN MENU OPTIONS}

\section{ENTRY}

This procedure is used to enter the manuscript into the tracking system. The 'entry' form is displayed and the user fills in everything except the BG number. The procedure computes the next BG number to be used and assigns it to the manuscript. The BG number is a seven-digit number; a three-character prefix, a hyphen, and a three-digit number with leading zeroes. The prefix (including the year digit) can be changed in the maintenance section. Authors should be entered with the last name first. Use upper- and lower-case letters for the authors and title. Valid entries for PROGram and TYPE of manuscript can be accessed during the entry process by pressing F1 when prompted to do so. The PROJect number must follow the following pattern: \#\#\#\#-\#\#\#\#-\#\#. A notation is made in the diary file, DIARYMS, that states "entered into system--to review committee". If DIARYMS does not exist, it is created the first time it is needed.

\section{QUERY/ARCH}

These two menu items have the same format, so they are discussed together. The QUERY selection is for active manuscripts; the $\mathrm{ARCH}$ selection is for archival manuscripts. The menu for each of these options has the following choices:

$\begin{array}{ll}\text { BG number: } & \text { Find manuscript(s) by BG number } \\ \text { Rpt number: } & \text { Find manuscript(s) by report number } \\ \text { Author: } & \text { Find manuscript(s) by author name } \\ \text { Project: } & \text { Find manuscript(s) by project number } \\ \text { Program: } & \text { Find manuscript(s) by program } \\ \text { Type: } & \text { Find manuscript(s) by type of manuscript } \\ \text { CTR: } & \text { List all manuscripts to CTR in given timeframe } \\ \text { List all: } & \text { List all manuscripts in database } \\ \text { Return: } & \text { Return to previous menu level }\end{array}$

When a query is performed the results are displayed on the screen. The BG number and Rpt number queries return only one manuscript as a result. The other queries may return more than one manuscript. If so, the user can scroll through the manuscripts by using the down arrow key. Long fields can be examined by pressing ALT F5. A report can be printed by pressing F7. The printed report does not have the same format as the form on the screen; it is compressed to put multiple manuscripts on one page. F2 should be pressed when the user is finished viewing the results. The results from the $B G$ number and report number queries can be printed by simply pressing the Print screen key on the PC.

\section{BG number}

This option allows the user to display a specific manuscript in the database. The user is prompted for the desired BG 
number. The user needs to enter the year digit, hyphen, and three digit number, (not the 'BG'). The result of this query is displayed on the screen in the 'routing sheet' format.

\section{Rpt number}

This option allows the user to display a specific manuscript, if it has been assigned an official report number. The user is prompted for the report number. The user needs to enter the entire report number, including prefix. The result of this query is displayed on the screen in the 'routing sheet' format.

\section{Author}

This option allows the user to retrieve all manuscripts by surname of the author or coauthor. There is a submenu asking which author(s) to retrieve.

Primary author: Additional authors: Either: Return: only main author

only search secondary authors Either main or secondary author Return to previous menu level

The user can scroll through the manuscripts that meet the criteria by using the down arrow key. Long fields can be examined by pressing ALT F5. A report can be printed by pressing F7. The printed report does not have the same format as the form on the screen; it is compressed to put multiple manuscripts on one page. F2 should be pressed when the user is finished viewing the results.

\section{Project}

This option allows the user to retrieve all manuscripts having a specific project number. The user can scroll through the manuscripts that meet the criteria by using the down arrow key. Long fields can be examined by pressing ALT F5. A report can be printed by pressing $\mathrm{F} 7$. The printed report does not have the same format as the form on the screen; it is compressed to put multiple manuscripts on one page. F2 should be pressed when the user is finished viewing the results.

The tracking system is set up so that new manuscripts have to be entered with a project number in this pattern: \#\#\#\#\#\#\#-\#\# All the project numbers from the original system on the Data General computer (DG) have the pattern \#\#\#\#-\#\#\#\#. The query retrieves manuscripts using either pattern. If the nine-digit number is given, all projects with those nine digits will be retrieved, regardless of the last two digits, if any.

\section{Program}

This option allows the user to retrieve all manuscripts with a 
specific program code. The user can scroll through the manuscripts that meet the criteria by using the down arrow key. Long fields can be examined by pressing ALT F5. A report can be printed by pressing F7. The printed report does not have the same format as the form on the screen; it is compressed to put multiple manuscripts on one page. F2 should be pressed when the user is finished viewing the results.

\section{Type}

This option allows the user to retrieve manuscripts that are a specific type of report. The user can scroll through the manuscripts that meet the criteria by using the down arrow key. Long fields can be examined by pressing ALT F5. A report can be printed by pressing F7. The printed report does not have the same format as the form on the screen; it is compressed to put multiple manuscripts on one page. F2 should be pressed when the user is finished viewing the results.

CTR

This option allows the user to retrieve all manuscripts that were sent "TO CTR" in a specific timeframe. The user enters a minimum and maximum date. Those dates are included in the query. The user can scroll through the manuscripts that meet the criteria by using the down arrow key. Long fields can be examined by pressing ALT F5. A report can be printed by pressing F7. The printed report does not have the same format as the form on the screen; it is compressed to put multiple manuscripts on one page and is grouped by program code. F2 should be pressed when the user is finished viewing the results.

\section{List all}

This option retrieves all manuscripts in the database and lists them on the screen in table form. All fields are not visible at one time; use the tab key or the right arrow to scroll across.

\section{UPDATE}

This selection allows the user to record movement of a manuscript from one location to another. The 'routing sheet' form is used. F2 should be pressed when the user is finished entering dates to indicate the manuscript has changed locations. After F2 is pressed the procedure interrogates the database and makes diary entries to indicate location changes. If a user updates a manuscript to a location before the one that was current when they chose this option, there will be no diary entry to reflect that activity. When a manuscript is returned with Director's Approval, the routine checks to see if the manuscript is an open File. If it is, a prompt is issued for the report number. The 'routing sheet' form currently has the following format: 
Review Commitee

To

From

\author{
Reviewers (all) \\ TPSG \\ Geologic Names Unit \\ Associate Branch Chief \\ CTR/Director's Approval \\ CTR (repro) \\ Ozalid Room \\ Clerical (copying) \\ CTR (OFSS) \\ Bibliography
}

\title{
REVIEW
}

This selection allows the user to perform all actions associated with reviewers. When reviewers are assigned an entry is made in a separate table that records the reviewers name and the type of manuscript. This allows a user to see the number and types of manuscripts all reviewers have reviewed. The following menu is displayed:

Assign reviewers: List reviewers: Add reviewers: Examine histogram: Return:
Assign reviewers to manuscript

List all reviewers for certain manuscript Assign additional reviewer(s) to manuscript Look at DB of reviewers Return to previous menu level

\section{Assign reviewers}

This selection allows the user to assign initial reviewers; reviewers cannot be added in this section. The procedure prompts for the BG no., then displays the author, title, and other information, and asks if this is the correct manuscript. If it is, the form for entry of reviewers names is displayed. The reviewer's name should be entered last name first. The reviewer's name is entered into the histogram or, if the reviewer's name already exists in the histogram, then just the code for the type of manuscript is added. The procedure will add to an existing entry only if the reviewer name already exists EXACTLY as entered. The table can hold 25 entries per reviewer name. This table can be purged and initialized in the MAINT section. An entry is made in the diary when reviewers are assigned.

\section{List reviewers}

This selection will list the reviewers for any manuscript. The user is prompted for the manuscript number (BG no.) and the reviewers are listed to the screen.

\section{Add reviewers}

This selection allows the user to add reviewers (one at a time) to a given manuscript. The same process that is used to 
assign initial reviewers is used to add names to the histogram. An entry is made in the diary when a reviewer is added.

\section{Examine histogram}

This selection allows the user to look at the histogram that contains the types of manuscripts each reviewer has reviewed. The histogram is set up to take 25 manuscripts per reviewer. This table should be reset at some interval (See MAINT section). The menu choices are:
Whole histogram:
Look at entire histogram
Look at one reviewer: List only one reviewer
Return:
Return to previous menu level

EDIT

This selection allows the user to alter information about any active manuscript. This has nothing to do with the location of the manuscript. The menu choices are:

$\begin{array}{ll}\text { Author: } & \text { Primary author } \\ \text { AddAuthor: Additional authors } \\ \text { Title: } & \text { Title } \\ \text { AddTitle: } & \text { Additional title } \\ \text { Type: } & \text { Manuscript type } \\ \text { Project: } & \text { Project number } \\ \text { Program: } & \text { Program }\end{array}$

A diary entry is made when information about the manuscript is edited.

\section{DELETE}

This selection allows the user to delete a manuscript from the active database. It does not delete the reviewers from the histogram. An entry is made in the diary when a manuscript is deleted.

\section{MAINT}

This selection contains maintenance features. The main menu has the following options:

\footnotetext{
Diary: $\quad$ Sort and rename current diary

Histogram: Sort and store current file and start new histogram

Reset: Unload: Edit the prefix/year and set number for $\mathrm{ms}$ to 1 Unload entire database to ASCII files Return: Return to previous menu level
} 


\section{DIARY}

The diary should periodically be cleared and the old file kept as a "history" file. The diary is an ASCII file named DIARYMS that is created and stored in the order that transactions are made (chronologically).

When the "Diary" selection is chosen the following actions take place:

a. The diary file is sorted by the BG number; a file named DIARYBG is created.

b. The original diary file (DIARYMS) is renamed with the current date as part of the name. The next time MSTRACK is executed, a new DIARYMS file will be created.

c. A message is displayed stating the names of the files to be printed (if user desires). To print the diary files the user must leave the application and return to DOs to print the diary. The diary cannot be printed through Paradox (PAL manual, version 3.0 , p. 347 \& PC PowerTools p. 845) because it generates the error "Insufficient memory to return to Paradox".

\section{HISTOGRAM}

Whenever the histogram table gets full or whenever otherwise desired the histogram table should be cleared and the old information stored as a "history" file. The histogram is stored as a paradox data table. It is created and stored in the order that reviewer's names are entered.

When the "Histogram" selection is chosen the following actions take place:

a. The HISTO table is sorted according to the last name of the reviewer. The original table is overwritten with the sorted table.

b. A PARADOX report is created from the HISTO table and named FRQ with the current date appended to the name.

c. The Paradox table HISTO is emptied of its records.

d. A message is displayed stating how to print the histogram information. To print the histogram information, the user needs to exit to DOS and print the report file.

\section{RESET}

At the beginning of each year (either fiscal or calendar, whichever system is being used), the four-character prefix used for the manuscript number (BG9-) needs to have the 'year' digit changed. The two letter code can also change, but the prefix must be exactly 4 digits. Also, the number sequencing is automatically reset to 1 when the prefix is changed. 


\section{Programmer's Documentation}

The lists below detail the components of the MSTRACK system.

Paradox Tables:

BIB - General information about active manuscripts and locations

REVIEW - Reviewers names and dates to/from--both active \& archival

JCTR - Dummy table used to copy reports and forms to CTR

CTR - Created by renaming ANSWER after query

BNUM - Contains next number to be used for manuscript number

RNUM - Contains prefix to be used for manuscript number

names

HISTO - Contains frequency distribution chart of reviewer

HIST2 - Used with HISTO

REPORTS - Contains Acceptable codes for type of report

PGMS - Contains acceptable codes for program

ARCH - Contains "archival" manuscripts

REF - Contains official bibliographic entries for

manuscripts

Miscellaneous files:

TRACKLB.LIB - created by TRKLIB, contains all procedures that make up the manuscript tracking system

MSTRACK. BAT - DOS file that invokes PARADOX with MENUMS1

DIARYMS - DOS file created first time needed; keeps record of activity in tracking system

Paradox PAL code:

MENUMS1.SC is the driver module; it displays the main menu shown on page 2. This is invoked when the user types MSTRACK.

TRKLIB.SC is the script that creates the procedure library TRACKLB. LIB. Before the tracking system is started, Paradox has to be invoked and this script "PLAYED" to create TRACKLB. LIB. The following procedures are contained in TRKLIB.SC

ENTRYMS1 - Initial Entry of manuscript into system

QMENU1 - Menu for types of queries/retrievals possible--

ACTIVE

QUBEG2 - Retrieval by BG number

QUREPT1 - Retrieval by official report number

QUAUTH2 - Retrieval by last name of author

QUPROJ1 - Retrieval by project number

QUPROG1 - Retrieval by program name

QUTYPE1 - Retrieval by type of report

QUCTR1 - Retrieval by date sent to CTR

QUALL1 - Retrieval of all manuscripts in active database

QARCH1 - Menu for types of queries/retrievals possible--

ARCHIVAL

QABEG2 - Retrieval by BG number 


\section{UNLOAD}

The other feature of the maintenance procedure is to unload the entire manuscript tracking system from Paradox into ASCII files that could be used to transfer the tracking data to another database management system. The UNLOAD selection creates ASCII files of all the Paradox tables that contain data. (There are several 'dummy' Paradox tables that are not included in the UNLOAD selection (JCTR and HIST2). They are used throughout the system for various reasons. These tables need to exist in Paradox for certain functions to take place properly, but in another DBMS, they may not be necessary.)

Table Name - - - - > ASCII file created

Bib
Bnum
Pgms
Reports
Review
Rnum
Histo
Arch
Ref

Bib

Bnum

Pgms

Reports

Review

Rnum

Histo

Ref

\section{BIB}

\author{
BIB. ASC \\ BNUM. ASC \\ PGMS. ASC \\ REP. ASC \\ REV. ASC \\ RNO. ASC \\ HISTO. ASC \\ ARCH. ASC \\ REF.ASC
}

This selection allows user to make a complete, official bibliographic entry for a manuscript, edit an existing bibliographic entry, or add the official report number. It does not allow this entry unless the manuscript is still active and has Director's approval. A manuscript cannot move to the archival database unless the bibliographic entry has been made.

\section{MOVE}

This sleection is used to move a manuscript from the active database to the archival database. The dates for Director's approval and bibliographic entry must exist. 


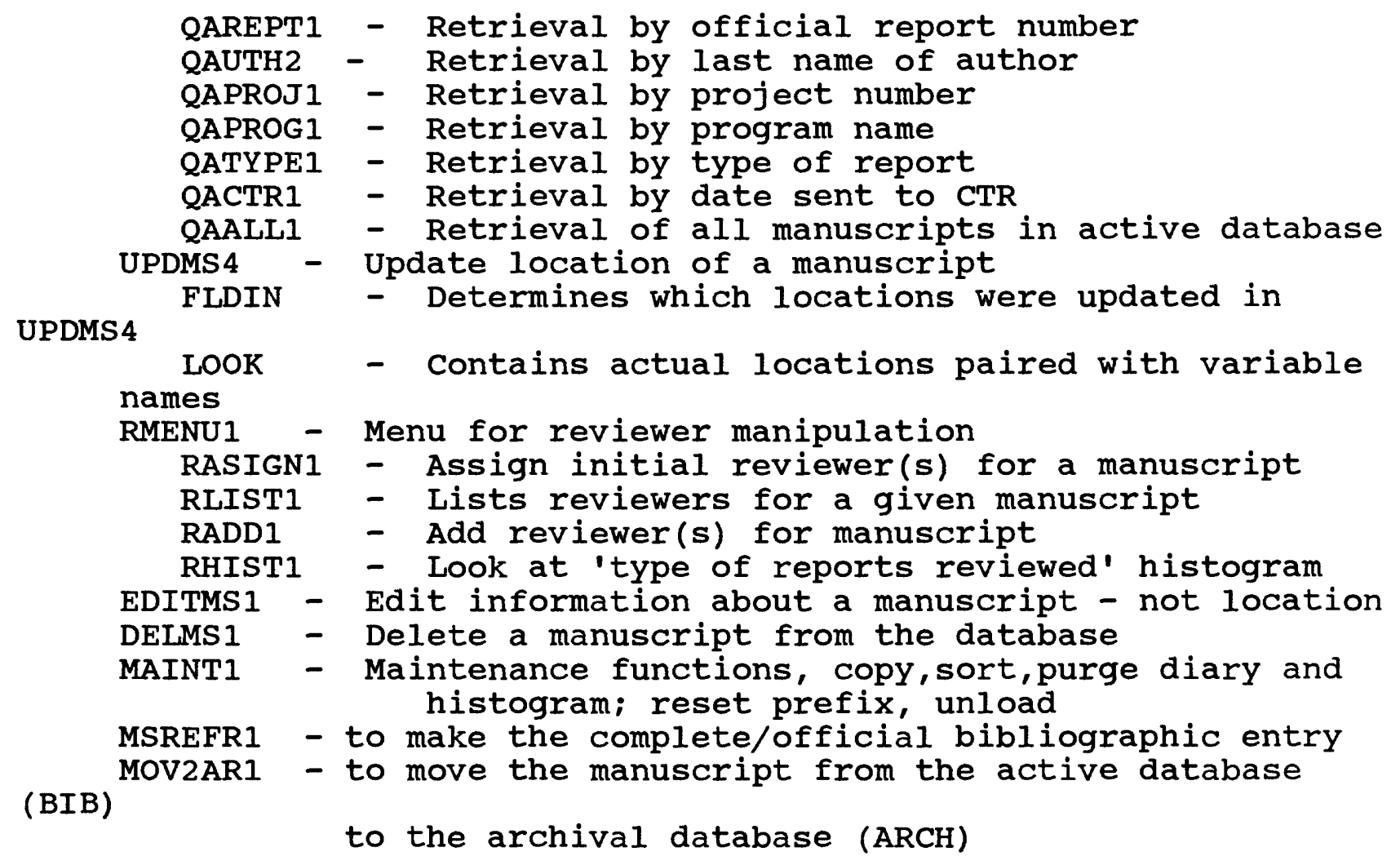

Al1 prompts for the BG number throughout the tracking system have the following format: "SUBMENU LOCATION: Enter BG number:" (example: "LOCATION UPDATE: Enter BG number: ") This is done so that the user can always tell in which sub-system they are working. The BG number should always be entered in the form FY-999 (example 1-012). Pressing the ENTER key at this prompt returns the user to the menu.

Every action that changes the database is recorded in a "diary file" named DIARYMS. The diary file is an ASCII file that is a chronological $\log$ of activity in the database. The entry made in DIARYMS has the date that the actual changes were made to the database, not the date that was entered into the database. 


\begin{abstract}
DESCRIPTION OF PROCEDURES
contained in TRKLIB.SC (TRACKLB.LIB)

(Refer to the description of main menu options for user

information)
\end{abstract}

\title{
ENTRYMS1
}

This procedure uses lookups from table BNUM (the prefix) and RNUM (last number used) and computes the next value for the numeric part of begno. Begno is a seven digit number; a three character prefix, a hyphen, and a three digit number with leading zeroes. A form is displayed and the user fills in everything except the BG number. A notation is made in the diary file DIARYMS that states "entered into system--to review committee" . If DIARYMS does not exist, it is created the first time it is referenced. validity checks on the table BIB are used: (1) lookup tables for program (table PGMS) and type of manuscript (table REPORTS), (2) picture \#\#\#-\#\#\#\#-\#\# for PROJect number.

\section{QMENU1/QARCH 1}

These two procedures are identical except for the tables they access, so they are discussed together. The Paradox QUERY command cannot accept variables for the table name, so two sections are required. QMENUI and the QU procedures are for active manuscripts; QARCHI and the QA procedures are for archival manuscripts. The main menu in each of these procedures has the following choices:

$\begin{array}{ll}\text { BG number: } & \text { Find manuscript(s) by BG number } \\ \text { Rpt number: } & \text { Find manuscript(s) by report number } \\ \text { Author: } & \text { Find manuscript(s) by author name } \\ \text { Project: } & \text { Find manuscript(s) by project number } \\ \text { Program: } & \text { Find manuscript(s) by program } \\ \text { Type: } & \text { Find manuscript(s) by type of manuscript } \\ \text { CTR: } & \text { List all manuscripts to CTR in given timeframe } \\ & \\ \text { Iist all: } & \text { List all manuscripts in database } \\ \text { Return: } & \text { Return to previous menu level }\end{array}$

The results of the query are displayed on the screen. The BG number and Rpt number queries return only one manuscript. The other queries may return more than one manuscript. If so, the user can scroll through the manuscripts by using the down arrow key. This technique for viewing the results of a query seems to work with one keystroke only when all the fields except the last one on the Paradox form are "displayonly"; if they are regular fields, the down arrow has to be pressed to go through each field.

Long fields can be examined by pressing ALT F5. A report can be printed by pressing F7. The printed report does not have the same format as the form on the screen; it is compressed to put multiple manuscripts on one page. F2 should be pressed when the user is 
finished viewing the results. The results from the BG number and report number queries can be printed by simply pressing the Print Screen key on the PC.

\section{QUBEG2/QABEG2}

This procedure allows the user to display a specific manuscript, in the appropriate database. The result of the query is displayed in the 'routing sheet' format. This routine does not use the QUERY command, but is placed in this section because it logically falls here.

\section{QUREPT1/QAREPT 1}

This procedure allows the user to display a specific manuscript, in the appropriate database, that has been assigned an official report number. The result of this query is displayed in the 'routing sheet' format. This routine does not use the QUERY command, but is placed in this section because it logically falls here.

\section{QUAUTH2/QAAUTH2}

This procedure allows the user to retrieve all manuscripts in the appropriate database by surname of the author or co-author (last name only). The submenu has the following form:

$\begin{array}{ll}\text { Primary author: } & \text { Only main author } \\ \text { Additional authors: } & \text { Only search secondary authors } \\ \text { Either: } & \text { Either main or secondary author } \\ \text { Return: } & \text { Return to previous menu level }\end{array}$

This information is listed to the screen one manuscript at a time. See information under QMENU1 above.

The technique used in Paradox to have forms and reports used by a table created during a query is explained in this paragraph. This technique is used in the author query, project query, program query, type query, and CTR query. The temporary ANSWER table is renamed to "CTR" so that it doesn't get overwritten inadvertently. The table "JCTR" has the exact same structure as the answer ("CTR") table. JCTR and its forms and reports exist so that the forms and reports may be copied to the table "CTR". Tables that get overwritten (like CTR does when ANSWER is renamed) get their forms and reports overwritten also. Since the ANSWER table doesn't have forms and reports, then the CTR table doesn't have forms and reports. That is why JCTR exists and its forms and reports are copied to CTR after the query is performed. The JCTR table and the table created by the query in any of the query modules QUAUTH2, QUPROG1, QUTYPE1, QUPROJ1, QUCTR1 must be exactly the same. That is why the JCTR table does not have a key item. To see the results of the query on the screen a 
FORM must be used. To see the results of the query on the printer a REPORT must be used.

The Custom Configuration program MUST be executed before these retrievals are made. In Paradox, the default order of variables in a query is table order; the change needs to be made in the Paradox Configuration file so that variables are returned in image order.

\section{QUPROJ 1/QAPROJ 1}

This procedure allows the user to retrieve all manuscripts in the appropriate database with a specific project number. These manuscripts are listed to the screen and can be printed on a report. See information under QMENU1 above.

\section{QUPROG1/QARROG1}

This procedure allows the user to retrieve all manuscripts in the appropriate database with a specific program code. The codes that are possible in the database for program are found in the table PGMS. The results of the query are listed to the screen and can be printed on a report. See information in QMENU1 above.

\section{QUTYPE1/QATYPE1}

This procedure allows the user to retrieve all manuscripts in the appropriate database with a specific type of report. The codes that are possible in the database for type of report are found in the table REPORTS. The results of the query are listed to the screen and can be printed on a report. See information in QMENU1 above.

\section{QUCTR1/QACTR1}

This procedure allows the user to retrieve all manuscripts in the appropriate database whose date in "LOC6IN" (to CTR) falls in a specified range. The dates given for minimum and maximum are included in the query request. This information is listed to the screen and can be printed to a report. See information in QMENU1 above. The printed report groups records by program.

\section{QUALL1/QAALL1}

This procedure allows the user to retrieve all manuscripts in the appropriate database and list them to the screen in table form. All fields are not visible at one time; use the tab key or the right arrow to scroll across. 


\section{UPDMS 4}

This procedure allows the user to record movement of a manuscript from one location to another. A 'routing sheet' form is used. LOC10OUT is the last location to be used; locs $11,12,13$, and 14 are not used at this time. Locl5in is the date of bibliographic entry. That date is entered through the bibliography selection, not the update selection.

When UPDMS4 is invoked it stores the last location that had a date when the update routine was entered (LASTVAR). After the update process is completed (Do_It!), the routine FLDIN interrogates the database and sees what locations (after LASTVAR) have dates. It makes the assumption that the users will not update to a location before the LASTVAR location. If a user does do that, there will be no diary entry to reflect that activity. The procedure uses the routine LOOK to attach names to the location positions and makes the entry in the diary. If loc6out (CTR/Director's Approval) was given a date, the routine FLDIN checks to see if the manuscript is an open file (OF). If it is, a prompt is issued for the report number. Report numbers for other types of manuscripts can be added in the bibliographic entry section (procedure MSREFR1).

\section{FLDIN}

This procedure is called by UPDMS4 after locations have been updated. The locations are updated during a WAIT RECORD, so UPDMS 4 does not know which variables have been changed. This routine checks all locations (variables) that occur after the last location that had a date when the update function was invoked. It checks all because some locations may be skipped. It checks from LASTVAR through locloout. Locs $11,12,13,14$ in/out are not currently used in this database and loclsin is a date for the bibliographic entry.

If a manuscript has been returned from CTR/Dir's approval (currently loc6out), the script checks to see if the report type is an open file (OF). If it is, then the user is prompted for the report number. This report number should be entered in its entirety (OF1-123).

\section{LOOR}

This procedure matches the database variables with the names of the 'actual' location. If the routing of the manuscripts changes - the following changes need to be made: (1) Change form F1 for BIB, both the description and the field (2) Change the script LOOK in TRKLIB.SC to agree with form FI for BIB. The only 'hard coded' reference to variable names is in QUCTR and UPDMS4 (where it starts looking backwards from LOCIOIN) 


\begin{tabular}{ll} 
LOC & \multicolumn{1}{c}{ Description } \\
1 & "Review Commitee" \\
2 & "Reviewers" \\
3 & "TPSG" \\
4 & "GNU" \\
5 & "ABC" \\
6 & "CTR"/Director's Approval" \\
7 & "CTR-repro" \\
8 & "Ozalid" \\
9 & "Clerical-copying" \\
10 & "CTR-OFss" \\
15 & "Bibliography"
\end{tabular}

\section{RMENU1}

This procedure displays the menu for reviewer manipulation.

Assign reviewers: Assign reviewers to manuscript

List reviewers: List all reviewers for certain manuscript

Add reviewers: Assign additional reviewer(s) to manuscript

Examine histogram: Look at DB of reviewers

Return: $\quad$ Return to previous menu level

\section{RASIGN1}

This procedure assigns initial reviewers; it cannot add reviewers. The procedure requests the BG no., opens the BIB table, displays the author, title, and other information and asks if this is the correct manuscript. If so, it retrieves the report type, and displays a form for entry of reviewers' names. The procedure enters reviewers' names into REVIEW table. Then it checks the HISTO table and adds a reviewer's name and type of manuscript or just adds the type of manuscript in the first available space MS1-MS25 to an existing entry. The procedure will add to an existing entry only if the reviewer already exists EXACTLY as entered. An entry is made in the diary when reviewers are assigned.

\section{RLIST1}

This procedure prompts for manuscript number (BG no.) and lists all reviewers for that manuscript.

\section{RADD1}

This procedure allows user to add reviewers ( 1 at a time) to a given manuscript. The same process that is used in RASIGN1 is used here to add names to the REVIEW table and add manuscript codes and reviewer's names to the HISTO table. When the manuscript is displayed and the banner reads "Press [F2] if this is the correct manuscript. Press [ESC] if this is NOT the correct manuscript", if the user presses ENTER, the next 
manuscript in the database is displayed. The up- and downarrows also work to look at different manuscripts in the database. An entry is made in the diary when a reviewer is added.

\section{RHI8T1}

This procedure allows user to look at histogram that contains the types of manuscripts each reviewer has reviewed. The HISTO table is set up to take 25 manuscripts. This table should be reset at some interval (See MAINT1). The menu choices are:

Whole histogram:

Look at entire histogram

Look at one reviewer: List only one reviewer

Return:

Return to previous menu level 


\section{EDITMS1}

This procedure allows the information about the manuscript to be changed. This has nothing to do with the location of the manuscript. The menu choices are:

$\begin{array}{ll}\text { Author: } & \text { Primary author } \\ \text { AddAuthor: } & \text { Additional authors } \\ \text { Title: } & \text { Title } \\ \text { AddTitle: } & \text { Additional title } \\ \text { Type: } & \text { Manuscript type } \\ \text { Project: } & \text { Project number } \\ \text { Program: } & \text { Program } \\ \text { None: } & \text { Don't wish to edit any of these }\end{array}$

A diary entry is made when information about the manuscript is edited.

\section{DELMS1}

This procedure deletes a manuscript from the active database (BIB). This does not delete the manuscript from the REVIEW table. An entry is made in DIARYMS when a manuscript is deleted.

\section{MAINT1}

This procedure contains maintenance features. The main menu has the following form:

Diary: Sort and rename current diary

Histogram: Sort and store current file and start new histogram

Reset:

Unload:

Edit the prefix/year and set number for ms to 1

Return:

Unload entire database to ASCII files

Return to previous menu level

The diary is an ASCII file named DIARYMS that is created and stored chronologically. When the "Diary" selection is chosen the following actions take place:

a. The diary file is sorted by the BG number; a file named DIARYBG is created. The DOS SORT command is accessed via the Paradox RUN command.

b. The file is renamed to be DIA + month + day + year. Month, day, year are obtained from Paradox functions. The next time MSTRACK is executed, the DIARYMS file is created.

c. If the user wants to print the diary files, they must leave the application and return to Dos to print the diary. The diary cannot be printed through Paradox (PAL manual, version 3.0, p. 347 \& PC PowerTools p. 845) because it generates the error "Insufficient memory to return to Paradox". 
The histogram is stored as Paradox data table HISTO. Periodically the histogram table should be purged and a new one started. When the "Histogram" selection is chosen the following actions take place:

a. The HISTO table is sorted using the Paradox SORT command by last name of the reviewer. The original table is overwritten with the sorted table.

b. A PARADOX report is created from the HISTO table and named FRQ + month + year (obtained from Paradox functions).

c. The Paradox table HISTO is emptied of its records. d. To print the histogram information, the user needs to exit to DOS and print the report file since the Paradox table is empty at this time. Before this selection is chosen the histogram can be printed through the REVIEWER selection of the main menu.

The "Reset" option allows the user to change the fourcharacter prefix used for the BG number (found in table BNUM) and automatically resets the manuscript sequencing number to 1 (found in table RNUM). The prefix must be exactly four digits.

The "Unload" option coverts the Paradox data tables into ASCII files that could be used to transfer the tracking data to another database management system. All tables that contain data are converted. There are several 'dummy' Paradox tables that are not included in the UNLOAD selection (JCTR and HIST2). These dummy tables are used throughout the system for various reasons. They tables need to exist in Paradox for certain functions to take place properly, but in another DBMS, they may not be necessary.

Table Name - - - - > ASCII file created

$\begin{array}{ll}\text { Bib } & \text { BIB. ASC } \\ \text { Bnum } & \text { BNUM. ASC } \\ \text { Pgms } & \text { PGMS. ASC } \\ \text { Reports } & \text { REP. ASC } \\ \text { Review } & \text { REV. ASC } \\ \text { Rnum } & \text { RNO.ASC } \\ \text { Histo } & \text { HISTO.ASC } \\ \text { Arch } & \text { ARCH. ASC } \\ \text { Ref } & \text { REF.ASC }\end{array}$

\section{MSREFR1}

This procedure allows user to make a complete, official bibliographic entry for a manuscript, edit an existing bibliographic entry, or add the official report number. It does not allow this entry unless the manuscript is still in the active database (Paradox table BIB) and has Director's approval. A manuscript cannot move from the active database (BIB) to the archival datbase ( $A R C H)$ unless the bibliographic entry has been 
made.

The bibliographic entry is made into Paradox table REF. The field DIRAPP is the same as the field LOC6OUT in the tables BIB/ARCH. The field BRANCHBIBLIO? is for use when software is developed to pull the information from REF for the branch bibliography. This will be a flag to indicate when a manuscript has already been included in a branch bibliography.

\section{MOV2AR1}

This procedure is used to move a manuscript from the active database (BIB) to the archival database (ARCH). The dates for Director's approval and bibliographic entry must exist. 


\section{Structure for Table BIB}

\begin{tabular}{|c|c|}
\hline Field Name & Field Type \\
\hline----------- & $\cdots-\cdots-\cdots$ \\
\hline Beg_no & $A 7 \star$ \\
\hline Author 1 & A50 \\
\hline Authorrem & A25 \\
\hline Type & A.2 \\
\hline Proj & A13 \\
\hline Prog & A 6 \\
\hline Report_no & A.10 \\
\hline Titlelong 1 & A25 \\
\hline Titlelong2 & A25 5 \\
\hline Loc 1 in & $\mathrm{D}$ \\
\hline Loc lout & $\mathrm{D}$ \\
\hline Loc 2 in & $\mathrm{D}$ \\
\hline Loc 2 out & $\vec{D}$ \\
\hline Loc 3 in & $\mathrm{D}$ \\
\hline Loc 3 out & $\mathrm{D}$ \\
\hline Loc 4 in & D \\
\hline Loc4out & $\mathrm{D}$ \\
\hline Loc 5 in & $\mathrm{D}$ \\
\hline Loc5out & $\mathrm{D}$ \\
\hline Loc 6 in & $\mathrm{D}$ \\
\hline Loc 6 out & $\mathrm{D}$ \\
\hline Loc 7 in & $\mathrm{D}$ \\
\hline Loc 7out & $\mathrm{D}$ \\
\hline Loc 8 in & $D$ \\
\hline Loc 8 out & $\mathrm{D}$ \\
\hline Loc 9 in & $\mathrm{D}$ \\
\hline Loc 9out & $\mathrm{D}$ \\
\hline Loc 10 in & $\mathrm{D}$ \\
\hline Loc 100 it & $\mathrm{D}$ \\
\hline Loc 15 in & D \\
\hline
\end{tabular}


Structure for Table ARCH

Field Name

Beg_no

Author 1

Authorrem

Type

Proj

Prog

Report_no

Titlelong1

Titlelong2

Loc 1 in

Loclout

Loc 2 in

Loc2out

Loc 3 in

Loc 3out

Loc 4 in

Loc 4 out

Loc 5 in

Loc5out

Loc 6 in

Loc 6 out

Loc 7 in

Loc7out

Loc 8 in

Loc 8 out

Loc 9 in

Loc 9out

Loc 10 in

Loc10out

Loc 15 in

Field Type

A 7*

A 50

A255

A2

A 13

A 6

A 10

A2 55

A2 55

D

D

D

D

D

D

D

D

D

D

D

D

D

D

D

D

D

D

D 


\section{Structure for Table RNUM}

$\begin{array}{ll}\text { Field Name } & \text { Field Type } \\ \text { Msnum } & \mathrm{N}\end{array}$

\section{Structure for Table BNUM}

Field Name

Begno
Field Type

A4 
Structure for Table REVIEW

\begin{tabular}{ll} 
Field Name & Field Type \\
\hline Beg_no & A7* \\
Rev1 & A30 \\
Rev2 & A30 \\
Rev3 & A30 \\
Rev4 & A30 \\
R1 to & D \\
R1bak & D \\
R2to & D \\
R2bak & D \\
R3to & D \\
R3bak & D \\
R4to & D \\
R4bak & D
\end{tabular}




\begin{tabular}{ll}
\multicolumn{2}{c}{ Structure for Table HISTO } \\
Field Name & Field Type \\
\hline Revr & $-A 30$ \\
Ms1 & A1 \\
Ms2 & A1 \\
Ms3 & A1 \\
Ms4 & A1 \\
Ms5 & A1 \\
Ms6 & A1 \\
Ms7 & A1 \\
Ms8 & A1 \\
Ms9 & A1 \\
Ms10 & A1 \\
MS11 & A1 \\
Ms12 & A1 \\
Ms13 & A1 \\
Ms14 & A1 \\
Ms15 & A1 \\
Ms16 & A1 \\
Ms17 & A1 \\
Ms18 & A1 \\
Ms19 & A1 \\
Ms20 & A1 \\
Ms21 & A1 \\
Ms22 & A1 \\
Ms23 & A1 \\
Ms24 & A1 \\
Ms25 & A1 \\
\hline
\end{tabular}

A5 
Structure for Table HIST2

\begin{tabular}{ll} 
Field Name & Field Type \\
\hline Revr & A30 \\
Ms1 & A1 \\
Ms2 & A1 \\
Ms3 3 A1 \\
Ms4 & A1 \\
Ms5 & A1 \\
Ms6 & A1 \\
Ms7 & A1 \\
Ms8 & A1 \\
Ms9 & A1 \\
Ms10 & A1 \\
MS11 & A1 \\
Ms12 & A1 \\
Ms13 & A1 \\
Ms14 & A1 \\
Ms15 & A1 \\
Ms16 & A1 \\
Ms17 & A1 \\
Ms18 & A1 \\
Ms19 & A1 \\
Ms20 & A1 \\
Ms21 & A1 \\
Ms22 & A1 \\
Ms23 & A1 \\
Ms24 & A1 \\
Ms25 & A1
\end{tabular}


structure for Table REPORTS

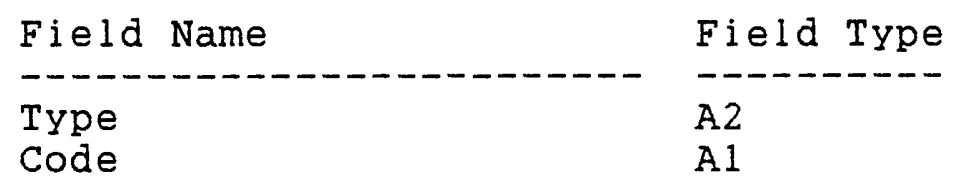

Structure for Table PGMS

Field Name Field Type

$\begin{array}{ll}\text { Type } & \text { A6 }\end{array}$ 
Structure for Table CTR

\begin{tabular}{ll} 
Field Name & Field Type \\
\hline Beg_no & A7 \\
Author1 & A50 \\
Authorrem & A255 \\
Type & A2 \\
Proj & A13 \\
Prog & A6 \\
Titlelong1 & A255 \\
Loc6in & D
\end{tabular}

Structure for Table JCTR

Field Name

Field Type

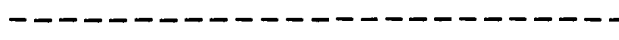

Beg_no

-

Author 1

A7

Authorrem

A 50

Type

A255

Proj

Prog

Titlelong 1

Loc 6 in

A2

A13

A 6

A255

D 
Structure for table REF

\begin{tabular}{ll} 
Field Name & Field Type \\
\hline Beg_no & - \\
Dirapp & A7* \\
Branchbiblio? & D \\
Bib1 & A1 \\
Bib2 & A255 \\
Bib3 & A255 \\
A25
\end{tabular}




\section{Form F1 for Bib table}

"Routing Sheet" form

Press [F2] when done or [esc] to abort

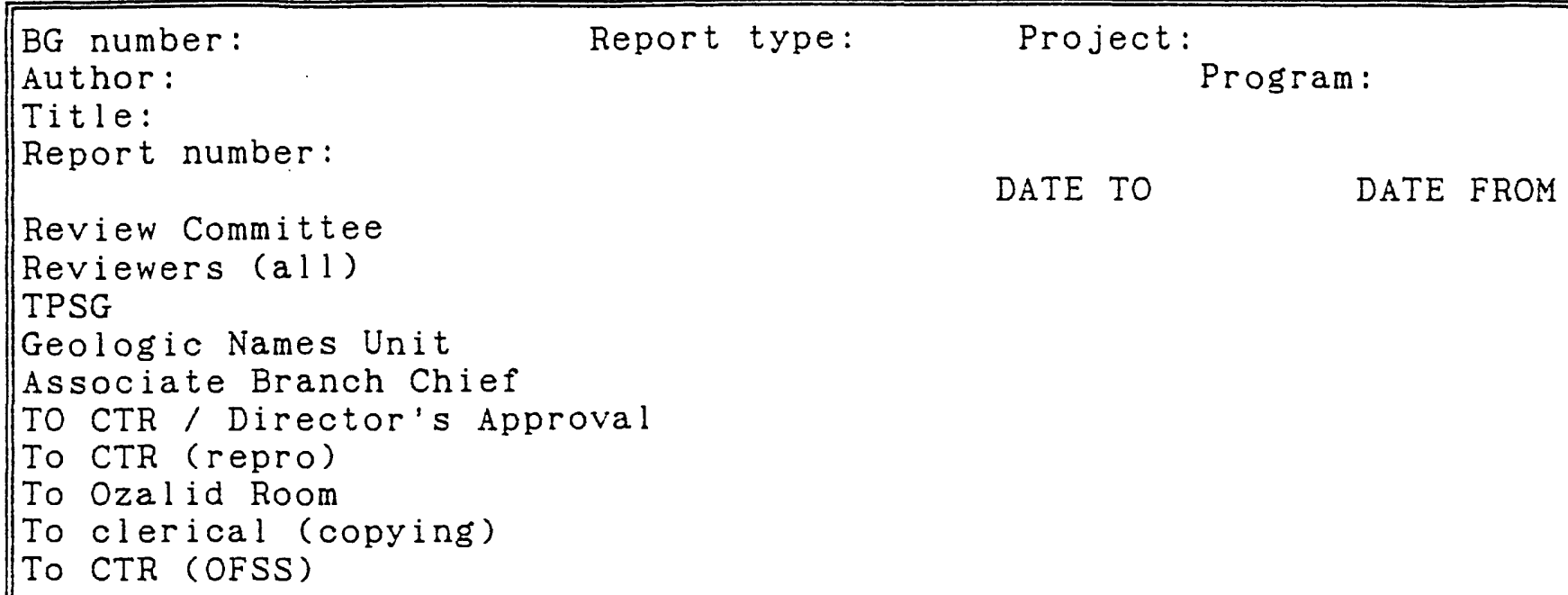


Author AddAuthor Title Additle Type Project Program None $* * * * * * * * * * * * * * * * * * * * * * * * * * * * * * * * * * * * * * * * * * * * * * * * * * * * * * * * * * * * * * * * * * * * * * * * * * * * * * * *$ * TECHNICAL PUBLICATIONS SUPPORT GROUP, BGC $10 / 4 / 90 *$ Edit Manuscript Information $* * * * * * * * * * * * * * * * * * * * * * * * * * * * * * * * * * * * * * * * * * * * * * * * * * * * * * * * * * * * * * * * * * * * * * * * * * * * * * * *$

* BG Number:

* Report type:

* Author:

* Additional Authors:

*

$*$

*

*

* Title:

*

*

*

*

*

* Additional Title:
Project number:

Program:

* 


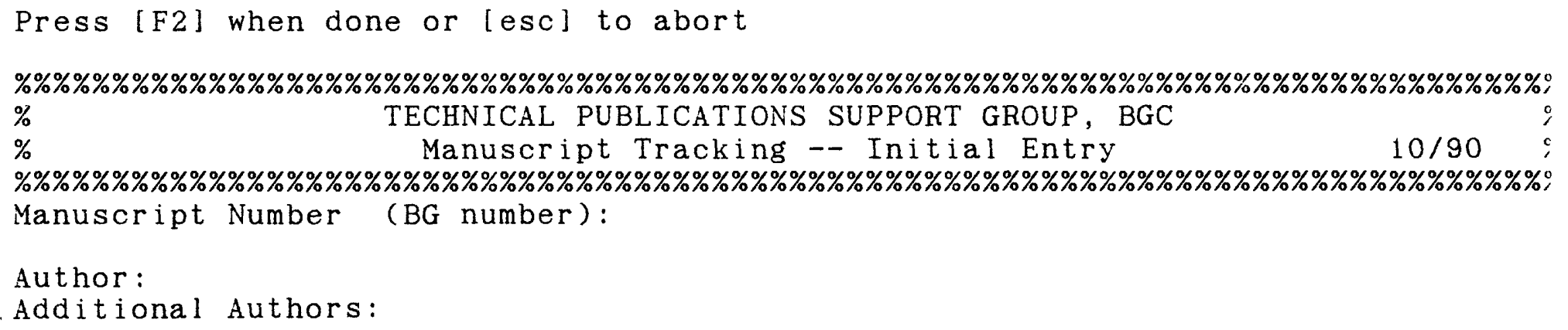


Form F4 for Bib table

(Form Fl from Review table embedded)
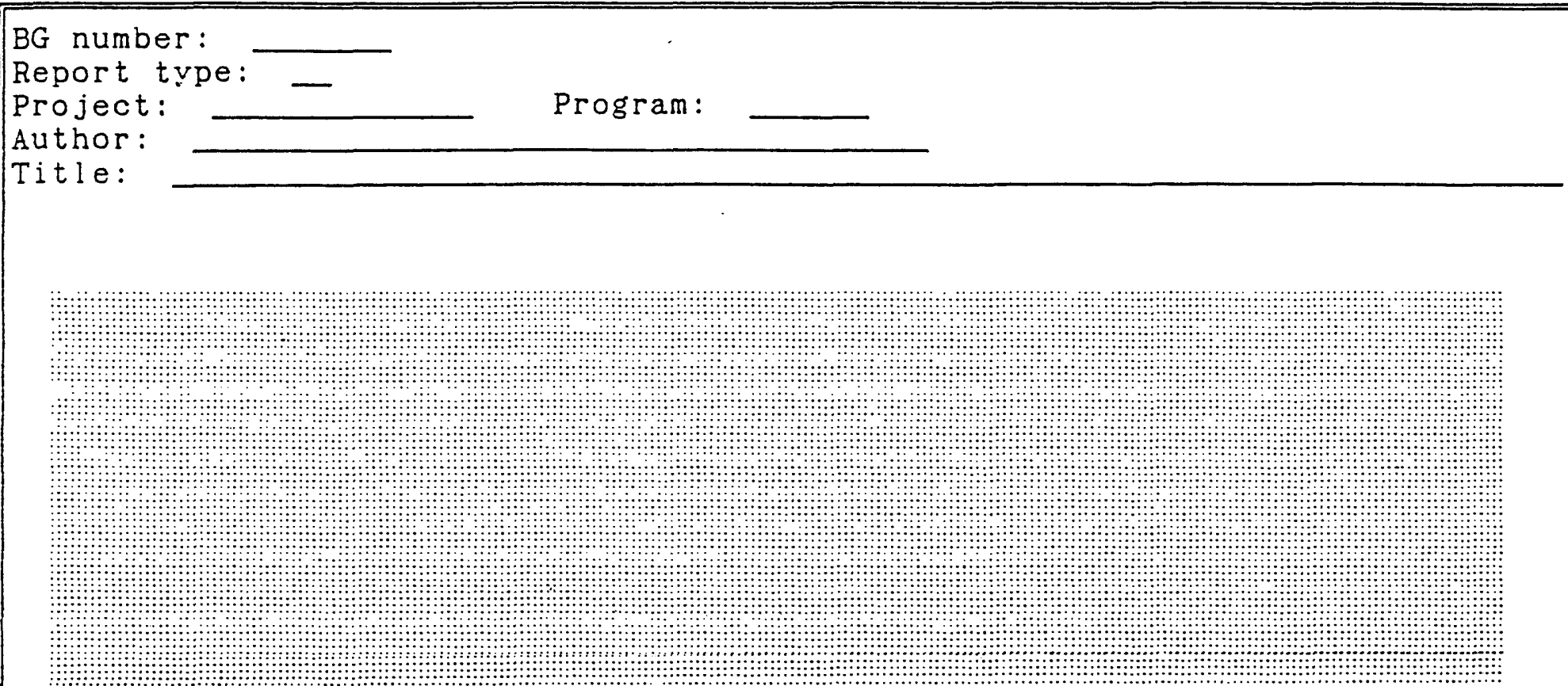

Form as it appears on screen

Press [F2] when done

BG number:

Report type:

Project:

Author:

Program:

Title: 
Form FI for Review table

(Embedded on Form F4 for Bib table)

Reviewers' Names

1st Reviewer:

2nd Reviewer :

3rd Reviewer :

4 th Reviewer:
Date sent to Reviewer

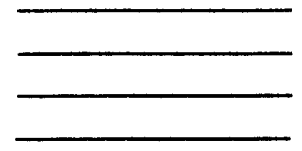


Form $F$ for Histo table

Multi-record form to display entire table

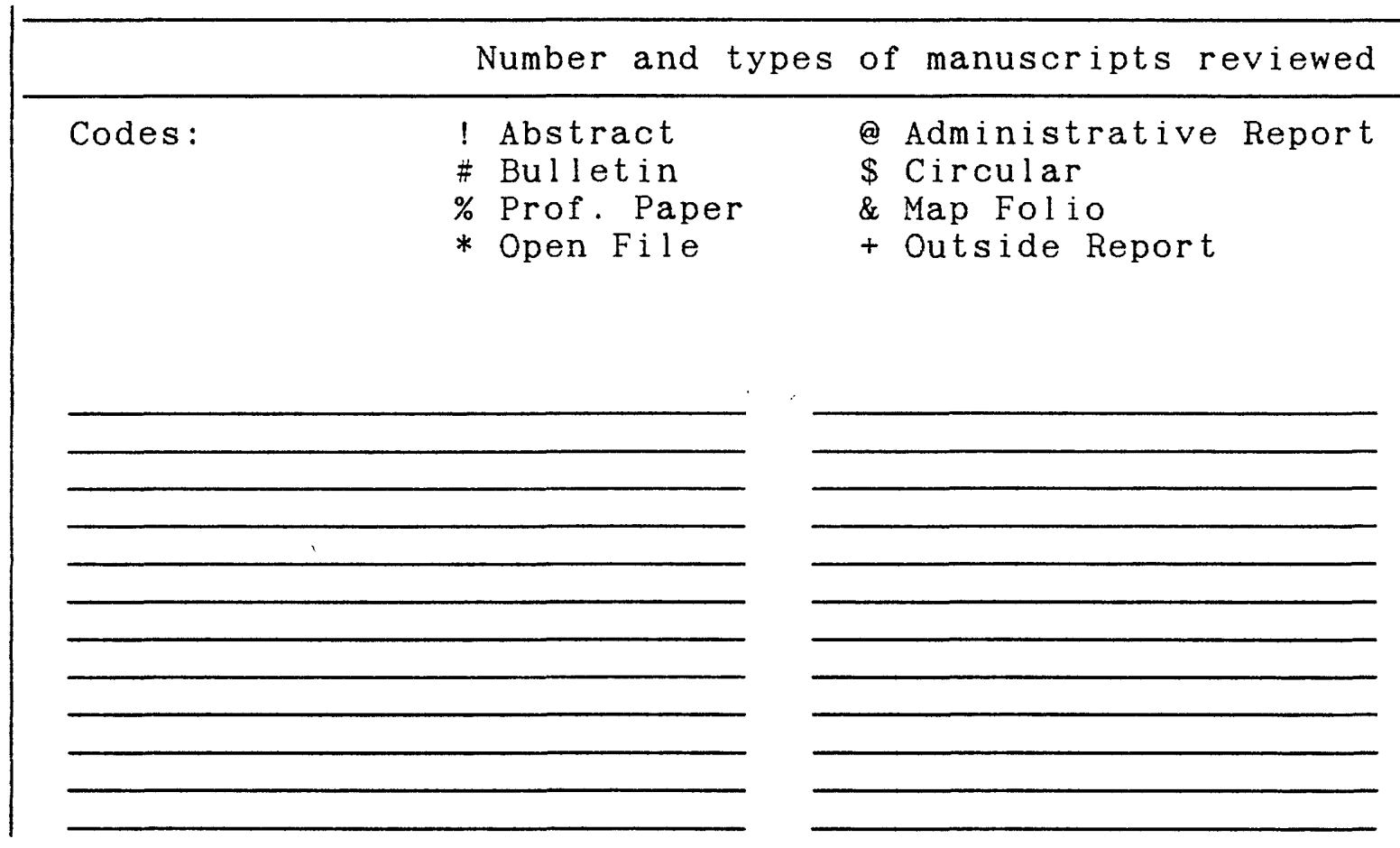


Form F1 for Histo table

\begin{tabular}{|c|c|c|c|}
\hline & Number and & $\begin{array}{l}\text { s of manuscripts } \\
\text { by }\end{array}$ & reviewed \\
\hline Codes: & $\begin{array}{l}! \text { Abstract } \\
\text { \# Bulletin } \\
\% \text { Prof. Paper } \\
\text { * Open File }\end{array}$ & $\begin{array}{l}@ \text { Administrative } \\
\$ \text { Circular } \\
\& \text { Map Folio } \\
+ \text { Outside Report }\end{array}$ & Report \\
\hline
\end{tabular}




\section{Form Fl for Jctr table}

\section{Manuscripts to CTR}

Type of report: — Project Number:

Program: Date to CTR:

Primary Author:

Additional Authors:

Title: 
Form F2 for Jctr table

Manuscripts by Project

Type of report:

Program:

Primary Author:

Additional Authors:

Title: 
Form F3 for Jctr table

Manuscripts by Program

Type of report:

Project:

Primary Author:

Additional Authors:

Title: 
Form F4 for Jctr table

Manuscripts by

requested author

Type of report: — Project Number:

Program:

Author:

Additional Authors:

Title: 
Form F5 for Jctr table

\section{Manuscripts by Type of manuscript}

\section{Program:}

Project:

Primary Author: Additional Authors:

Title: 
Report R2 for Jctr table

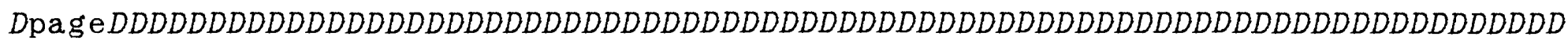
$\mathrm{mm} / \mathrm{dd} / \mathrm{yy}$

Manuscripts by project

Page 99؛

AAAAAAAAAAAAA

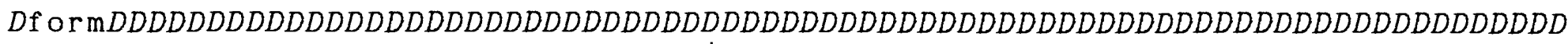

AAAAAAA

Report type: AA Program: AAAAAA

Author: AAAAAAAAAAAAAAAAAAAAAAAAAAAAAAAAAAAAAAAAAAAAAAAAAA

other authors: AAAAAAAAAAAAAAAAAAAAAAAAAAAAAAAAAAAAAAAAAAAAAA

Tit le: AAAAAAAAAAAAAAAAAAAAAAAAAAAAAAAAAAAAAAAAAAAAAAAAAAAAAAA

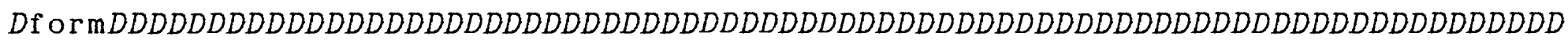

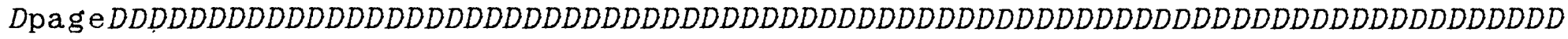

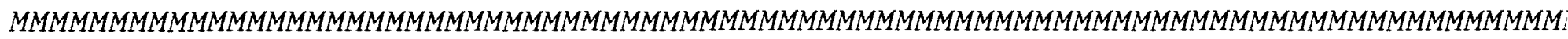


Report R3 for Jctr table

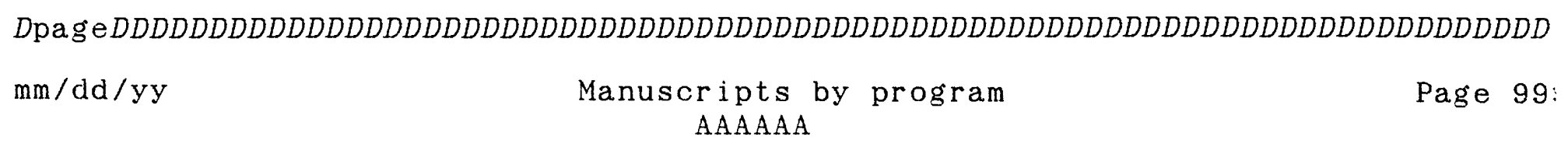

Tit le: AAAAAAAAAAAAAAAAAAAAAAAAAAAAAAAAAAAAAAAAAAAAAAAAAAAAAAA

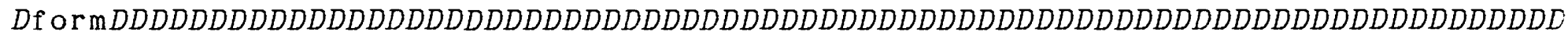

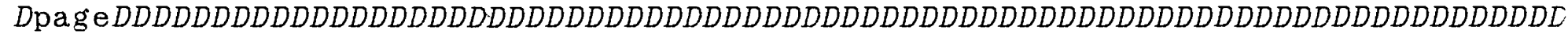
MMMMMMMMMMMMMMMMMMMMMMMMMMMMMMMMMMMMMMMMMMMMMMMMMMMMMMMMMMMMMMMMMMMMMMMMMMMMMMM. 
Report R4 for Jctr table

Dpage $D D D D D D D D D D D D D D D D D D D D D D D D D D D D D D D D D D D D D D D D D D D D D D D D D D D D D D D D D D D D D D D D D D D D D D D D D D$ $\mathrm{mm} / \mathrm{dd} / \mathrm{yy}$

Manuscripts by requested author

Page 999

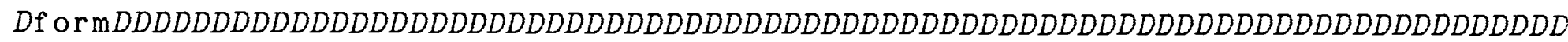

AAAAAAA Report type: AA Project: AAAAAAAAAAAAA Program: AAAAAA Author: AAAAAAAAAAAAAAAAAAAAAAAAAAAAAAAAAAAAAAAAAAAAAAAAAA other authors: AAAAAAAAAAAAAAAAAAAAAAAAAAAAAAAAAAAAAAAAAAAAAA

Tit le: AAAAAAAAAAAAAAAAAAAAAAAAAAAAAAAAAAAAAAAAAAAAAAAAAAAAAA

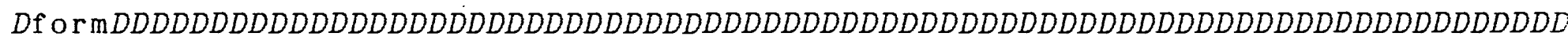

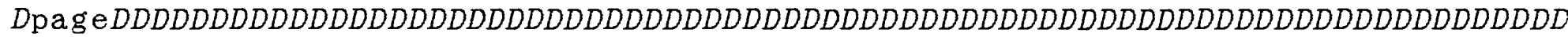
MMMMMMMMMMMMMMMMMMMMMMMMMMMMMMMMMMMMMMMMMMMMMMMMMMMMMMMMMMMMMMMMMMMMMMMMMMMMMMM 
Report R5 for Jctr table

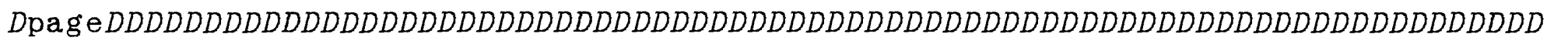

mm/dd/yy Manuscripts by type of manuscript Page 999 $\mathrm{AA}$

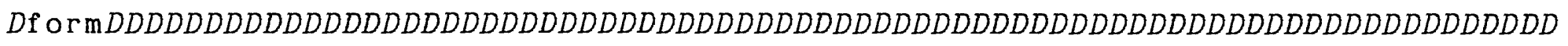

AAAAAAA Program: AAAAAA Project: AAAAAAAAAAAAA

Aut $\mathrm{t}$ or: AAAAAAAAAAAAAAAAAAAAAAAAAAAAAAAAAAAAAAAAAAAAAAAAAA

Other author $s$ : AAAAAAAAAAAAAAAAAAAAAAAAAAAAAAAAAAAAAAAAAAAAAA

Tit $l$ e: AAAAAAAAAAAAAAAAAAAAAAAAAAAAAAAAAAAAAAAAAAAAAAAAAAAAAAA

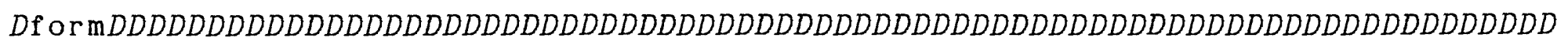

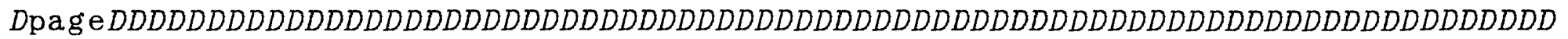

MMMMMMMMMMMMMMMMMMMMMMMMMMMMMMMMMMMMMMMMMMMMMMMMMMMMMMMMMMMMMMMMMMMMMMMMMMMMMMMI 


\begin{abstract}
Dpage $D D D D D D D D D D D D D D D D D D D D D D D D D D D D D D D D D D D D D D D D D D D D D D D D D D D D D D D D D D D D D D D D D D D D D D D D D D$ $\mathrm{mm} / \mathrm{dd} / \mathrm{yy}$

Manuscripts to CTR

Page $99 \varsigma$

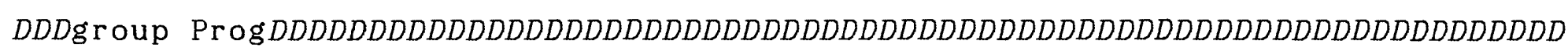

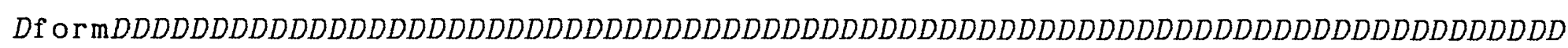
AAAAAAA Type of report: AA Program:
Project Number:
Date to CTR:
AAAAAAAAAAAAA
$\mathrm{mm} / \mathrm{dd} / \mathrm{yy}$

Primary Author: AAAAAAAAAAAAAAAAAAAAAAAAAAAAAA

Add i t ional Authors: AAAAAAAAAAAAAAAAAAAAAAAAAAAAAAAAAAAAAAAAA

Tit l e: AAAAAAAAAAAAAAAAAAAAAAAAAAAAAAAAAAAAAAAAAAAAAAAAAAAA

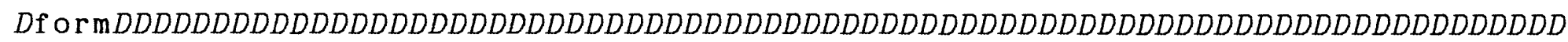

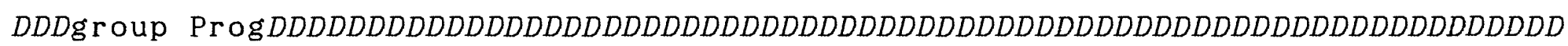

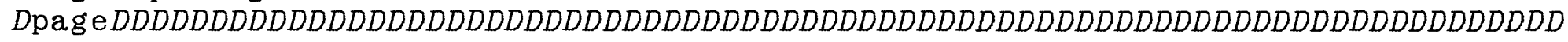


Report R1 for Histo table

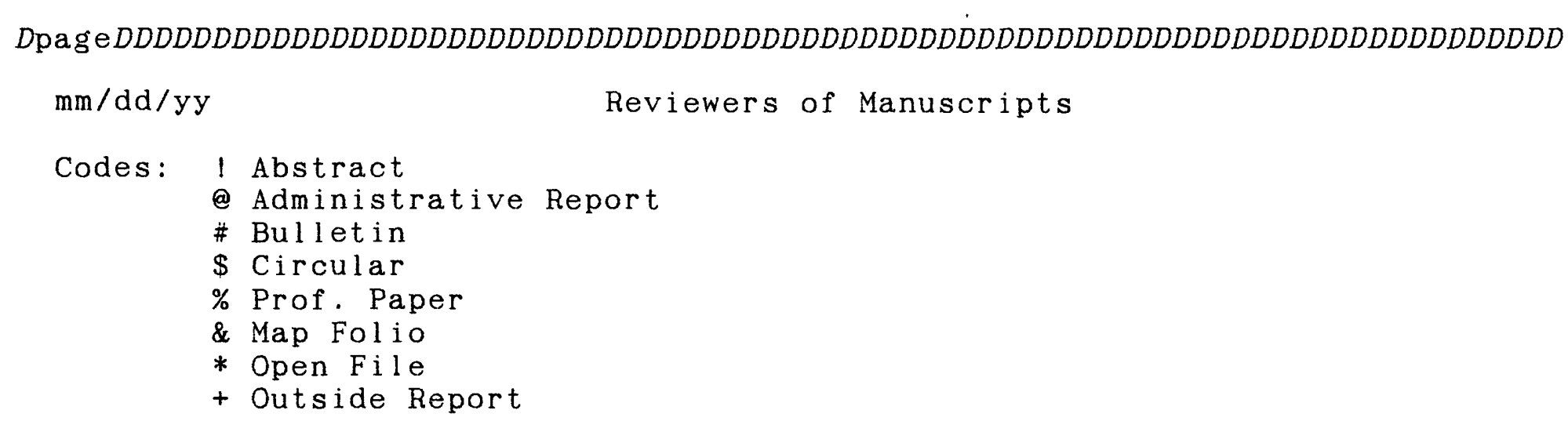

